\title{
Low frequency pulsar observations with the international LOFAR station FR606
}

\author{
Louis Bondonneau ${ }^{1}$, Jean-Mathias Grießmeier ${ }^{1,2}$, Gilles Theureau ${ }^{1,2,3}$ \\ and Maciej Serylak ${ }^{4}$ \\ ${ }^{1}$ Laboratoire de Physique et Chimie de l'Environnement et de l'Espace LPC2E \\ CNRS-Université d'Orléans, 45071 Orléans Cedex 02, France \\ email: louis.bondonneau@cnrs-orleans.fr \\ ${ }^{2}$ Station de Radioastronomie de Nançay, Observatoire de Paris, CNRS/INSU, 18330, France \\ ${ }^{3}$ Laboratoire Univers et Théories LUTh, Observatoire de Paris, CNRS/INSU, Université Paris \\ Diderot, 5 place Jules Janssen, 92190 Meudon, France \\ ${ }^{4}$ SKA SA, Cape Town, South Africa
}

\begin{abstract}
The pulsar's signal passes through the interstellar medium (ISM) which leads to both chromatic dispersive delays and multipath pulse broadening. These effects have a strong frequency dependence $\left(\mathrm{f}^{-2}\right.$ and $\mathrm{f}^{-4}$ respectively). Pulse profiles of pulsars are also frequencydependent leading to some degeneracy with the ISM imprint. Furthermore, many pulsars show a turnover of their spectrum around $\sim 100 \mathrm{MHz}$. For all these reasons, the frequency band below $100 \mathrm{MHz}$ contains a lot of information about both the pulsar emission and the ISM. Our study is based on a LOw Frequency ARray (LOFAR) monitoring campaign using the international station FR606. Firstly, we demonstrate the importance of a monitoring campaign. Secondly, we calculate median spectra and locate the turnover frequency for 3 pulsars $(B 0809+74$, B1133+16, B1508+55).
\end{abstract}

\section{LOFAR single station observations}

International LOw Frequency ARray (LOFAR) stations can be used as individual telescopes ("local mode" observations) and provide data sets complementary to those obtained with the LOFAR Core. Due to the flexibility of international stations, this mode is particularly adapted to monitoring observations (observations with weekly cadence). FR606 has $\sim 13 \%$ of the sensitivity of the LOFAR core, but in return it is much more flexible. Single station observations currently represent $\sim 40 \%$ of the observing time. Since 2014, FR606 participates in a programme of pulsar monitoring with the LOFAR HBA (High Band Antenna 110-190 MHz) ( 100 pulsars) and conduct another monitoring programme of 15 pulsars with the LOFAR LBA (Low Band Antenna 25-90 $\mathrm{MHz}$ ), described in Grießmeier et al. (2017).

\section{Flux calibration and variations}

Low frequency observations are strongly affected by short-term variations of the observed flux density due to scintillation in the interstellar medium. For this reason, the flux measured in a single observation is not fully representative of the flux of the observed pulsar. This effect combined with residual instrumental bias make the validation of the calibration difficult (Bilous et al. (2016), Kondratiev et al. (2016)). For this reason, a $50 \%$ error bar is used to encompass these short-term variations (Bilous et al. (2016)). We use the median to obtain the pulsar's intrinsic spectra (see purple line in Fig. 1). In these spectra, the frequency turnover is well-defined. 


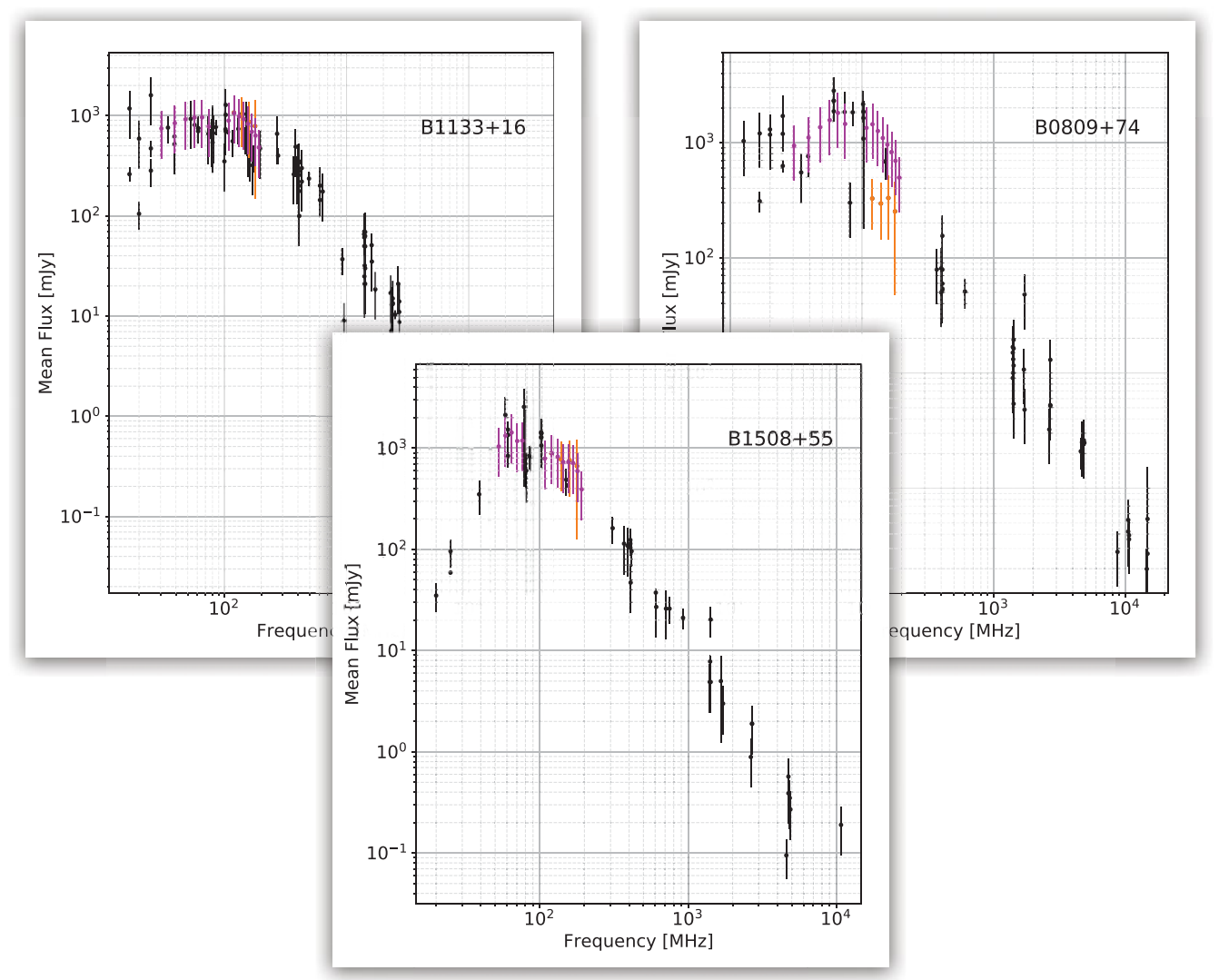

Figure 1. Spectra of 3 pulsars. Orange dots: LOFAR core observation, Bilous et al. (2016). Black dots: archival data (see Bilous et al. (2016) and references therein). Purple dots: median spectra with $50 \%$ error bars from $\sim 15$ LBA observations and $\sim 100$ HBA observations with FR606.

\section{Results}

We have calculated robust fluxes versus the scintillation (median on more than 100 observations for HBA and $\sim 15$ observations for LBA through years of data). Combined with previous observations (Bilous et al. (2016) and references therein) we have been able to produce well defined median spectra and increase the sensitivity on the location of the spectral turnover.

\section{Conclusions and perspectives}

Low frequency monitoring observations are highly efficient to study the ionised interstellar medium. This study is a first step to increase the sensitivity on the spectrum to detect the spectral turnover.

\section{References}

Bilous et al. 2016, A\&A, 591, A134

Griessmeier et al. 2017, Proccedings IAU Symposium, No. 337, submitted (this volume)

Kondratiev et al. 2016, A\& $A$, 585, A128 